\title{
Difusión de los resultados en salud de los hospitales
}

\section{Diffusion of hospital health outcomes}

\section{J. Gost Garde}

Cada vez un mayor número de organizaciones, tanto públicas como privadas, desarrollan propuestas para mejorar el nivel de información de los servicios sanitarios. Junto a las ya clásicos de la Agency for Healthcare Research and Quality (AHRQ), el Canadian Institute for Health Information (CIHI), el Australian Council on Healthcare Standards (AHCS), el Performance Assessment Framework for Hospitals (PAtH) de la oficina europea de la $\mathrm{OMS}^{1}$, cabe mencionar especialmente al Choose and Book del National Health Service ${ }^{2}$ por cuanto su objetivo es facilitar la elección de hospital y servicio sanitario a sus ciudadanos.

En España, la Sociedad Española de Calidad Asistencial (SECA) ha desarrollado un conjunto de indicadores de calidad ${ }^{3}$ que permite realizar tareas de benchmarking entre los hospitales. Desde la iniciativa privada hay que citar al ya clásico TOP 20 de IASSIST ${ }^{4}$ con 15 años de andadura. Por lo que respecta al Sistema Nacional de Salud, además de los indicadores globales que facilita el ministerio ${ }^{5}$, cada servicio de salud ha elaborado su propio sistemas de información lo que dificulta la comparación entre los mismos.

Recientemente algunos artículos en revistas biomédicas han abordado la relación entre la reputación y algunas dimensiones de la actividad hospitalaria ${ }^{6-8}$.

La difusión en diciembre de 2014 del I Monitor de Reputación Sanitaria (MRS) ${ }^{9}$ que cuenta con el aval del Monitor Empresarial de Reputación Corporativa (MERCO) que incluye, entre otras, una clasificación de los servicios hospitalarios con mejor reputación por especialidad, de los hospitales en función de sus servicios y de los médicos por especialidad $^{10}$, ha tenido una importante repercusión pública. Su principal novedad es que integra una evaluación objetiva (indicadores de calidad y rendimiento asistencial), una subjetiva (percepción de profesionales médicos, enfermeros, pacientes y periodistas) y una evaluación directa de méritos reputacionales realizada por técnicos propios.

En este número de Anales del Sistema Sanitario de Navarra se publica el artículo del profesor Mira y colaboradores, "La reputación de los

Ex-jefe del Servicio de Medicina Preventiva y Calidad Hospitalaria del Complejo Hospitalario de Navarra.
Correspondencia: Javier Gost Garce gardkost@orange.es 
hospitales. Bases para el desarrollo de un índice de reputación de los hospitales"11 cuyo objetivo es analizar los atributos que debiera reunir un índice de reputación de los hospitales españoles.

No es objeto de esta editorial el poner de manifiesto las ventajas y limitaciones de las metodologías utilizadas en los diferentes estudios, sino en abogar por la necesidad de desarrollar y poner en marcha un sistema de información que sea accesible y útil al conjunto de los ciudadanos y que no se convierta ni en un ejercicio académico ni en un producto publicitario.

En un contexto de libre mercado sanitario, donde la elección del hospital depende principalmente de las preferencias de los ciudadanos (y de su seguro y/o de sus posibilidades económicas, obviamente), parece haberse evidenciado que la elección sigue estando priorizada por factores tales como la distancia y accesibilidad a la que se encuentra el hospital, la recomendación de su médico o su reputación ${ }^{12,13}$ más que por la comparación de indicadores objetivos ${ }^{14,15}$. Abundando en lo anterior, existen estudios que sugieren una relación directa entre la calidad de las webs corporativas de los hospitales -que están diseñadas para reforzar la reputación del centro, satisfacer las expectativas de los consumidores e influir en su decisión de compra- con la posición que ocupa el hospital ${ }^{16}$. Asimismo no puede obviarse el hecho de que la información requerida por los pacientes varía de acuerdo con sus características socio-culturales y demográficas ${ }^{17,18}$.

Sin embargo, en nuestro país y en la práctica diaria, los consumidores tenemos opiniones que, con frecuencia, solo están basadas en experiencias o impresiones personales, de familiares o amigos, sin datos objetivos en los que fundamentarlas. Además nuestra capacidad de elegir, a pesar del marco teórico legislativo, es más bien escasa. Salvo circunstancias excepcionales acudimos al hospital que tenemos asignado o al que nos remite nuestro médico.

Si estas premisas son ciertas, ¿para qué necesitamos la información? Y en el supuesto de que dicha información sea necesaria ¿cómo debería ser presentada?

Preguntas tales como: ¿Qué y cuánto se hace? ¿Por qué y para qué se hace? ¿Con qué criterios? ¿Con qué resultados? ¿A qué coste? debieran tener respuesta en una información accesible e inteligible para la generalidad de los ciudadanos, incluidos -no nos olvidemos-los gestores y los profesionales sanitarios. Porque, ¿de qué sirve conocer que un hospital ha obtenido una puntuación de 10.000 y otro de 3.500 si se interpreta como que el primero ha sacado matrícula de honor y el segundo ha suspendido?

Teniendo en cuenta además que la variabilidad en la práctica clínica es un hecho incontestable ${ }^{19}$, la necesidad de aportar a los ciudadanos una información sobre los resultados en salud de los hospitales, es una exigencia de trasparencia que debiera ser una obligación ética, cuando no legal, del Sistema Nacional de Salud.

Por ello propugnamos que todos los hospitales financiados total o parcialmente con cargo a presupuestos públicos, deben hacer públicos sus resultados, incluidos los de satisfacción y -como no- también los económicos. Y ello a pesar de las reticencias de determinados políticos y gestores a su difusión ${ }^{20}$. 
Ésta es una actuación necesaria pero no suficiente. El Ministerio de Sanidad está obligado a elaborar y difundir una información agregada que posibilite la comparación de hospitales y servicios. Utilizando criterios e indicadores comunes y consensuados entre todos los servicios de salud de las comunidades autónomas (previo los ajustes de riesgo que fueran necesarios). Es un tema complejo al que debiera darse respuesta sin mayor demora.

¿Con qué objetivos? Para los usuarios debe posibilitar la libertad de elección informada del centro sanitario. Para los políticos y gestores debe ser un instrumento imprescindible para la toma de decisiones, evitando disfunciones flagrantes entre los centros y servicios sanitarios.

¿Cómo debería ser presentada? De tal manera que además de su utilidad, se garantice su accesibilidad. De una manera unívoca. Que la búsqueda de la información no requiera navegar por un mar proceloso, con sirenas que nos confundan y nos impidan o retrasen en nuestra búsqueda.

La utilización de herramientas basados en la web es un fenómeno imparable (aunque no hay que olvidar que un importante número de personas no tienen acceso o no utilizan internet). No deben escatimarse esfuerzos para desarrollar una herramienta que dé respuesta a las dificultades existentes, algunas de las cuales ya se han comentado.

Solamente un sistema basado en la trasparencia permite decisiones libres e informadas, a todos los niveles. $Y$ en esta tarea debemos exigirnos y comprometernos todos.

\section{BIBLIOGRAFÍA}

1. http://www.pathqualityproject.eu/ Acceso en mayo 2015.

2. http://www.nhs.uk/choiceintheNHS/Yourchoices/appointment-booking/Pages/about-the-referral-system.aspx. Acceso en mayo 2015.

3. http://www.calidadasistencial.es/imagen/gestion/biblioteca/335.pdf. Acceso en mayo de 2015.

4. http://www.iasist.com.es/es/top-20. Acceso en mayo 2015

5. http://www.msssi.gob.es/estadEstudios/estadisticas/sisInfSanSNS/home. htm. Acceso en mayo 2015

6. Navarro IM, Mira JJ, Lorenzo S. Desarrollo y validación de un cuestionario para medir la reputación social de los hospitales. Gac Sanit 2012; 26: 444-449.

7. Mira JJ, Lorenzo S, NaVARRo I. Hospital reputation and perceptions of patient safety. Med Princ Pract 2014; 23: 92-94.

8. Jung K, Feldman R, Scanlon D. Where would you go for your next hospitalization. J Health Econ 2011; 30: 832-841.

9. http://www.merco.info/es/posts/361. Acceso en mayo 2015.

10. Trilla A, Prat A, Asenjo MA, Guinovart C, Bertran MJ, Llach M. Análisis de la reputación de los hospitales españoles: relación con su producción científica en cuatro especialidades. Med Clín 2006; 126: 768-770.

11. Mira JJ, Lorenzo S, Navarro IM, Guilabert M, Pérez-Jover V. La reputación de los hospitales españoles. Bases para el desarrollo de un índice de reputación. An Sist Sanit Navar 2015; 38: 247-254.

12. KetelaAr NA, Faber MJ, Braspenning J, Westert GP. Patients' expectations of variation in quality of care relates to their search for comparative performance information. BMC Health Serv Res 2014; 14: 617.

13. Mosadeghrad AM. Patient choice of a hospital: implications for health policy and management. Int J Health Care Qual Assur 2014; 27: 152-164. 
14. Ketelaar Na, Faber MJ, Westert GP, Elwyn G, Braspenning JC. Exploring consumer values of comparative performance information for hospital choice. Qual Prim Care 2014; 22: 81-89.

15. http://www.slideshare.net/slideshow/embed_code/42254029. Acceso en mayo 2015

16. Ford EW ${ }^{1}$, Huerta TR, Diana ML, Kazley AS, Menachemi N. Patient satisfaction scores and their relationship to hospital website quality measures. Health Mark Q 2013; 30: 334-348.

17. Laverty AA ${ }^{1}$, Dixon A, Millett C. Do patients' information requirements for choice in health care vary with their socio-demographic characteristics? Health Expect 2013; 28. doi: 10.1111/hex.12086 